
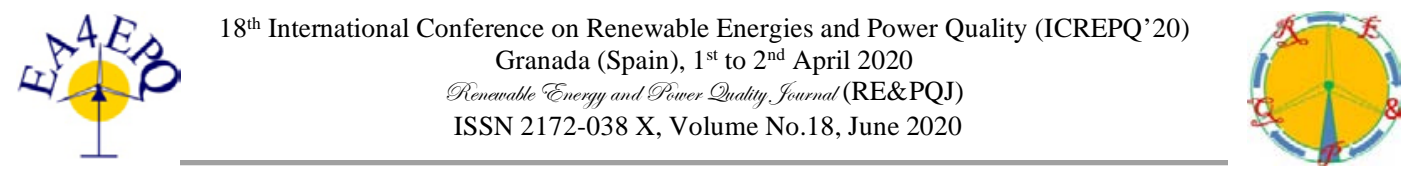

\title{
Design of a photovoltaic solar plant: Distributed generation in medium tension to a bar of the electricity substation.
}

\author{
O. Cabeza-Gras, and V. Jaramillo-García \\ Department of Physics and Earth Sciences \\ University of A Coruña \\ Campus da Zapateira s/n. 15071 A Coruña (Spain) \\ Phone/Fax number:+0034 981 167000, e-mail: oscar.cabeza@udc.es, vladimir.jaramillog@udc.es
}

\begin{abstract}
Usage of not conventional and renewable energy sources (NCRE), as photovoltaic plants, to generate electricity and self-consumption of residential areas, shops and industry, is known as distributed generation. The present research aims to design a photovoltaic solar plant of $1.5 \mathrm{MW}$ to be ground mounted. It will be interconnected with the bar of the substation of the local distributer of electricity in medium tension. The project is explained from its beginning, including site description of the terrain chosen, solar irradiation data, distribution and inclination of solar panels, electrical connexion among the panels, and inter-connection with the local distributer. The calculated production of the planned solar plant would be near 2.5 million of $\mathrm{kWh}$, having taken into account the technical loss of energy in about $3.5 \%$ including all loss sources. Finally, economic study has been carried out to obtain a return period for the investment of only two years, with an estimated profit, in the 20 years of the solar plant life, of about 15 million USD. In conclusion, it is possible technical and economically to invest in this solar plant, but it would be also desirable for ecological and social reasons.
\end{abstract}

Keywords. Energy, photovoltaic plant, solar irradiation, Arenilla district (Ecuador).

\section{Introduction}

Solar irradiation that reach the Earth surface is composed by two components, direct and diffuse (part of this last comes from reflected irradiation in the earth, air and clouds) [1]. As it is well known, this radiant energy can be transformed in electricity taking advantage of the photovoltaic effect. For that, many photovoltaic panels connected among them can produce enough energy to be competitive with other dirty technologies using fossil fuels [2]. Another advantage is that solar plants can be near the centres of consumption because they are versatile in placement, and no contaminant for the environment once installed. do not generate greenhouse effect gases (GHG) during the operation, and also, they do not contaminate water or air. In addition, they are noiseless during operation [3]. Thus, this technology is fundamental to develop the electrical distributed generation.

The actual mix of electrical generation in Ecuador is dominated by hydroelectricity, which represents nearly 90 $\%$ of the production. The remaining $10 \%$ corresponds to thermal using fossil fuel (7.5 \%) and only a $2.5 \%$ to no conventional. This mix is clean because it does not emit GHGs, but it is still considered as conventional due to the energy production distribution [4]. Actual circumstances of climate alert need the evolution of the conventional electric network toward a smart one, this will allow a bidirectional interchange of energy and information, integrating the removable generation of small and medium sizes with the main web taking or giving energy on demand. This is one of the main aims of the distributed generation paradigm, which include control elements which allow to operate the net and to solute technical problems much faster [5].

Ecuador has regions with high values of solar irradiation as El Oro province, with presents a maximum and minimum potential of electricity generations of 5.0 and $3.6 \mathrm{kWh} / \mathrm{m}^{2}$ each average day. The province of El Oro is placed in the south of Ecuador, very far from the electricity production centres, also it is far from the National Transmission System (SNT) optimal charge point, and in the extremal of the distribution network. These facts do that the electricity product is of low quality and shortages and tension lowering are not unusual. Smart distribution networks would solve these problems, going from a static and unidirectional gestion to another one dynamic and bidirectional [6]. In addition, the planned solar plant will contribute to the local development and industrialization because it generates important improvements in the electrical distribution system, increasing the quality and comfort of the electricity supply and assuring its stability [7].

To conclude, the main aim of this communication is to design a photovoltaic solar plant ground mounted in Arenillas district, of El Oro province of Ecuador, to transform the solar radiation in electricity. The objectives are to demonstrate it is viable technically and economically, giving the corresponding valuation index. 


\section{Localization of the site, modelling and climate data}

After some inspections in the El Oro province, we have chosen a site in the Arenillas district that is near an existing electrical substation, it is plane and it does not have any water source. The area chosen to install the solar plant is shown in Figure 1. The place has as coordinates $\mathrm{X}=603509,847$ and $\mathrm{Y}=9607771,608$ in UTM WGS 84, zonw $17 \mathrm{~S}$ and it has an altitude of $30 \mathrm{~m}$ over sea level. Then we proceed to determine the Solar Irradiation by using the METEONORM software.

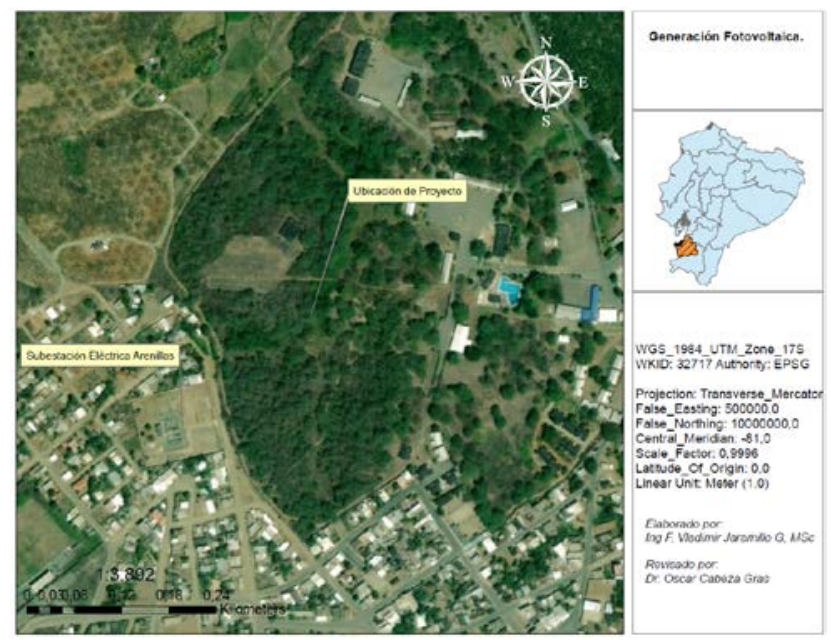

Figure 1. Location of the site chosen to mount the photovoltaic plant.

In Figure 2 we show the global irradiation along the average year. It is about $2,126 \mathrm{kWh} / \mathrm{m}^{2}$.year, direct one $1,205 \mathrm{~kW} / \mathrm{m}^{2}$ year and diffuse radiation is 921 $\mathrm{kWh} / \mathrm{m}^{2}$ year. It is observed that in the area the solar irradiation is stable along the twelve months, having its maximum in March and October, and minimum in July, but differences are below a $25 \%$ of solar irradiation.

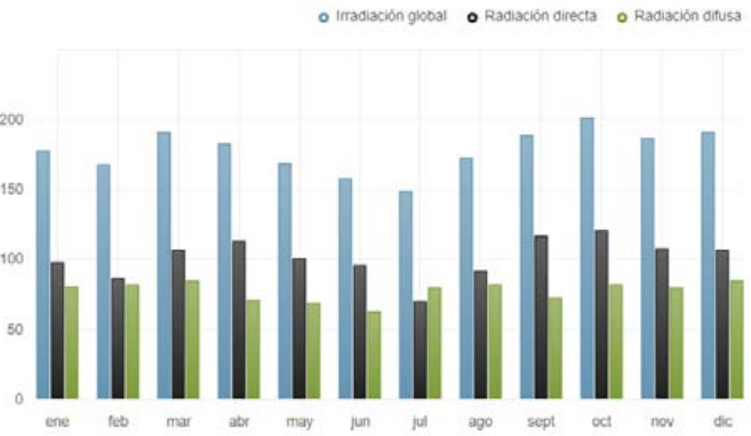

Figure 2. Global irradiation (blue), diffuse (green) and direct (black) during an average year.

The selected site is not exposed to obstacles that could produce shadows, as mountains or trees. This information is resumed in the solar chart with far obstacles, given in Figure 3, where we can observe the horizon line and the sun inclination land along all year months, as observed the site has not shadows. As it was expected from figure 2, the longest days are in March and October, and the shortest ones in May and July. In any case differences are small because its proximity to the equatorial line.

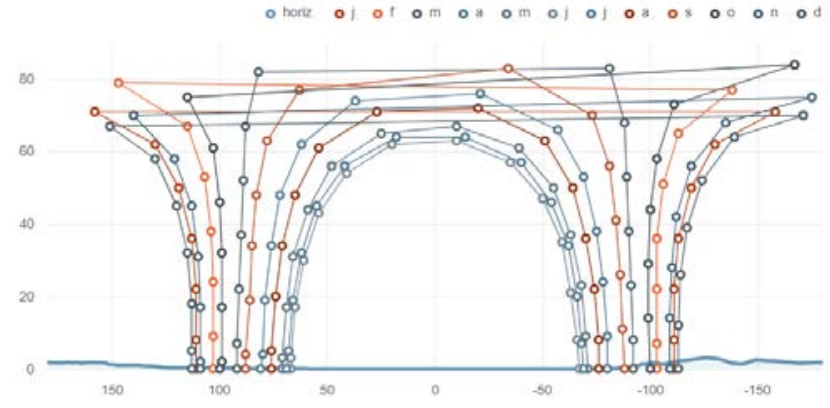

Figure 3. Solar chart of the site including far obstacles.

We can conclude that the solar irradiation to the horizontal land is very similar taking into account the obstacles, thus its presence represents only a $0.25 \%$ of energy loss in the calculus. Thus, we can conclude that the optimum solar radiation in the site chosen are, per year: Global $=2,121$ $\mathrm{kWh} / \mathrm{m}^{2}$ from which direct $=1,202 \mathrm{kWh} / \mathrm{m}^{2}$ (56.5 \%) and diffuse $919 \mathrm{kWh} / \mathrm{m}^{2}$, (43.5 \%).

By using a 3D model, we place the site geographical location of the exact place where we want to install the photovoltaic plant. For that we have used se Geographical Information System (GIS). Results obtained, including altitude over the see level, is presented in Figure 4. With this data we can calculate the exact surface area of our site, resulting in 60,888 $\mathrm{m}^{2}$ as observed in Figure 4.

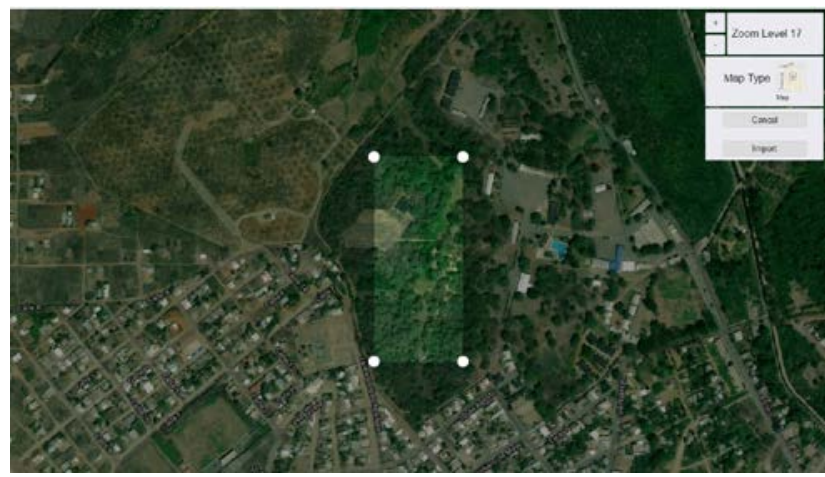

Figure 4. Geographical localization of the site chosen to install the photovoltaic plant proposed.

After that, we performed an analysis of the terrain inclination using the 3D model for the level curves. In addition, we must delimitate the effective area where the solar panels will be placed, which has an area of 44.184 $\mathrm{m}^{2}$. So, we have space enough to install the solar plant, and it could grow in the future. Then, we proceed with the levelling of the site, as observed in Figure 5. Fortunately, our terrain is plain so levelling is vey small, which is positive for our plant mounting and operation. 


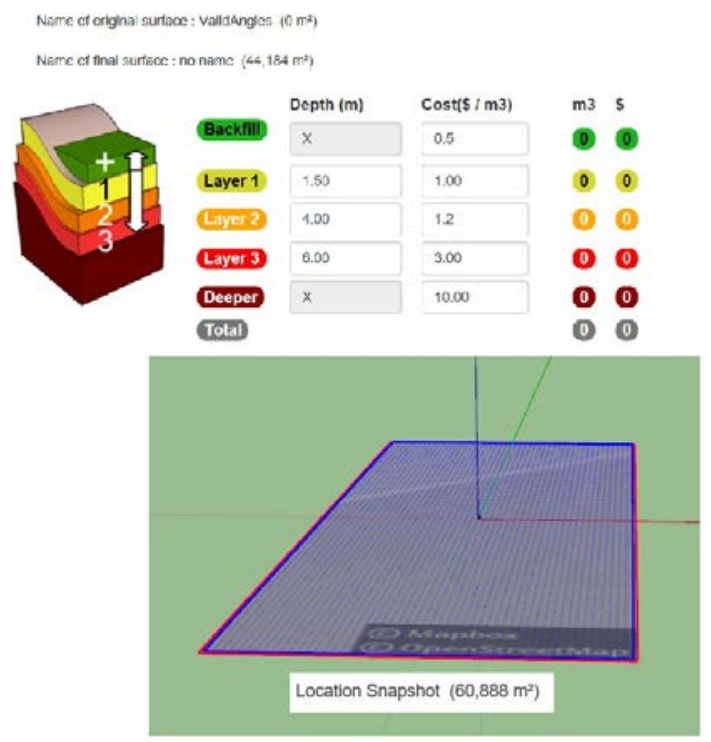

Figure 5. Levelling of the site surface.

\section{Solar plant design}

Now, we have used a special software to design the solar plant, and thus, with its aid we will choose the most suitable photovoltaic panel (trade and model), its dimensions, technology used and nominal power. The characteristics of the chosen panel are shown in Table I.

Table I. - Type of Panel

\begin{tabular}{|c|c|c|}
\hline Manufacture & Model & Dimensions (cm) \\
\hline Upsolar ShangHai & UP-M255P & $99.2 \times 164 \times 4$ \\
\hline
\end{tabular}

It is poly-crystalline silicon with a nominal power (Wp) of $255 \mathrm{~W}$ and single face. This panel is mounted in an aluminium structure inclined 25 degrees with land. We have chosen this model for being ideal to ground mounted and because it has a very high power and relative low cost. We will need 5,882 panels to reach a nominal power of 1.5 MW.

The geometry of the placement of this panel ground mounted appears in Figure 6 below

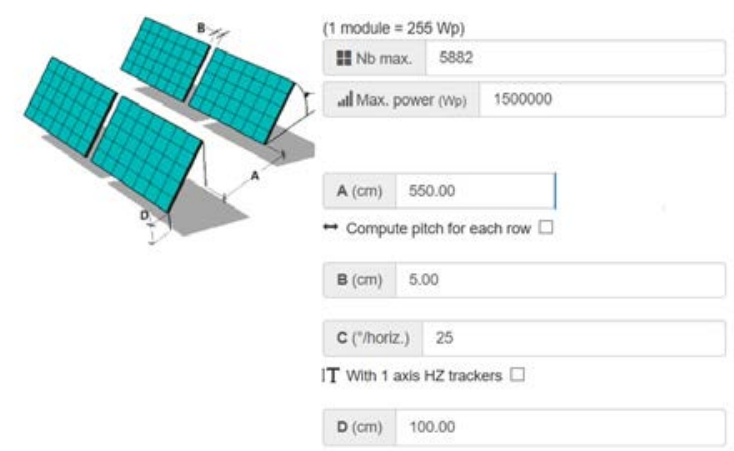

Figure 6. Geometry of the placement of the solar panels in the site chosen.

In Figure 7 we show the attachment points of the panels to the metal structure, which must be enough strong to resist the wings (not vey hard in this area) but as smaller as possible to cover the minimum of active surface of the panel.

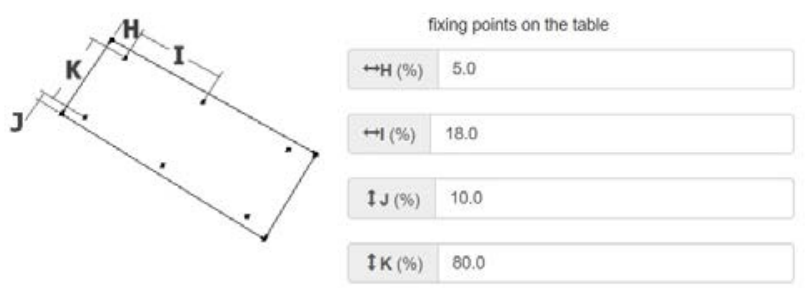

Figure 7. Attachment points of the panels to the structure.

Now, we proceed to extrapolate that data to the overall field chosen to place the solar plant to reach the planned nominal power of $1.5 \mathrm{MW}$. The final disposition of the 5,882 panels is observed in Figure 8, where we observe that forms about 50 lines formed by 117 panels each.

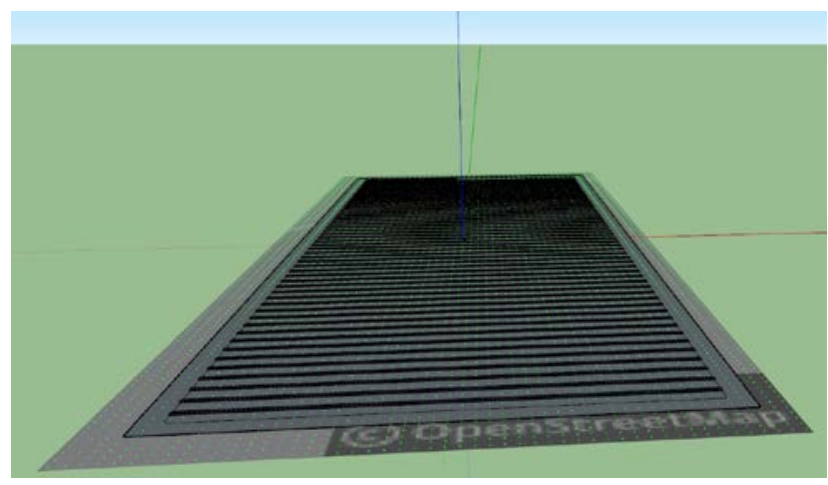

Figure 8. Photovoltaic solar plant in a 3D virtual representation formed by 5,882 solar modules

Now, we must configurate the inverters taking into account the number and type of the installed solar modules. Results obtained with the named software are shown in Figure 9.

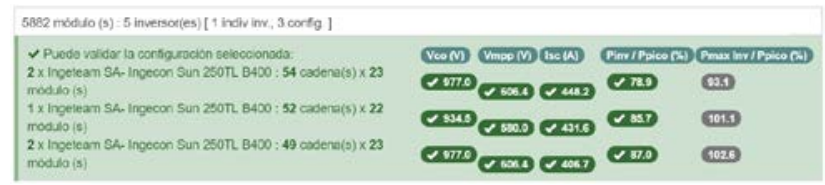

Figure 9. Configuration and number of the inverters used.

Finally, the chosen solution to interconnect the panels is the "S" network type, creating an electrical diagram whose general structure is shown in Figure 10. All panels are connected in five series of about 1200 solar panels each, as observed in Figure 11.

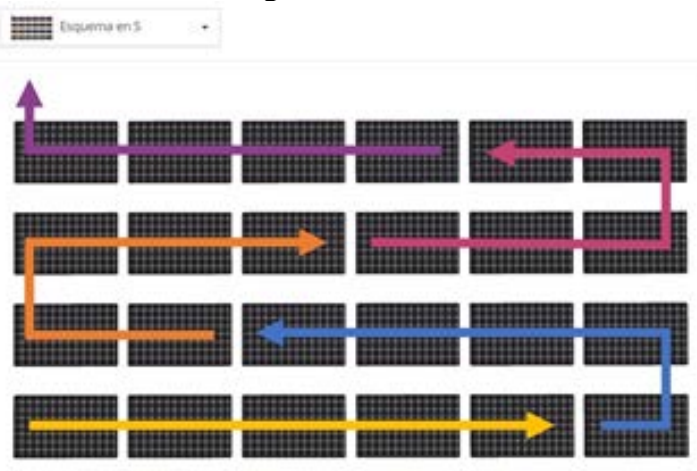

Figure 10. S disposition to connect solar panels. 


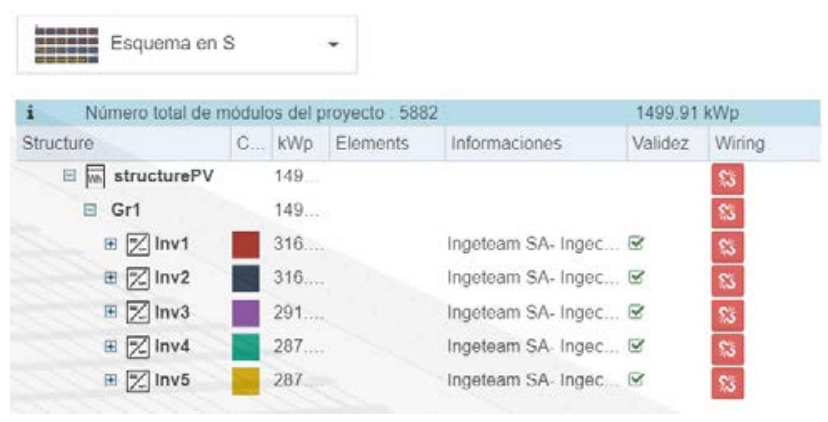

Figure 11. Electrical connections among solar modules in S shape and the number of inverters planned.

Respecting the electrical loss, we calculate about a $2.0 \%$ due to dust in the panel surface, about $9.4 \%$ due to the high temperature, about a $2.8 \%$ due to reflexion (at the beginning and the end of the day). Also, we must consider that the panel decreases efficiency with time, so a degradation factor of the panel of about $5.1 \%$ estimated for a period of 10 years. In addition, we must consider the technical loss of the produced electricity due to dc wires (0.6\%), inverters $(2.1 \%)$ and ac wires $(0.5 \%)$.

\section{Interconnection between the solar plant and the $69 \mathrm{kV}$ bar of the substation.}

To interconnect the solar plant and the $69 \mathrm{kV}$ bar to the Arenillas electrical substation, we use a wire of only $84 \mathrm{~m}$ length, type ACAR 500 MCM. In Figure 12 we show the map of the area with the solar plant site, the electrical substation location and the proposed electrical cable connecting both.

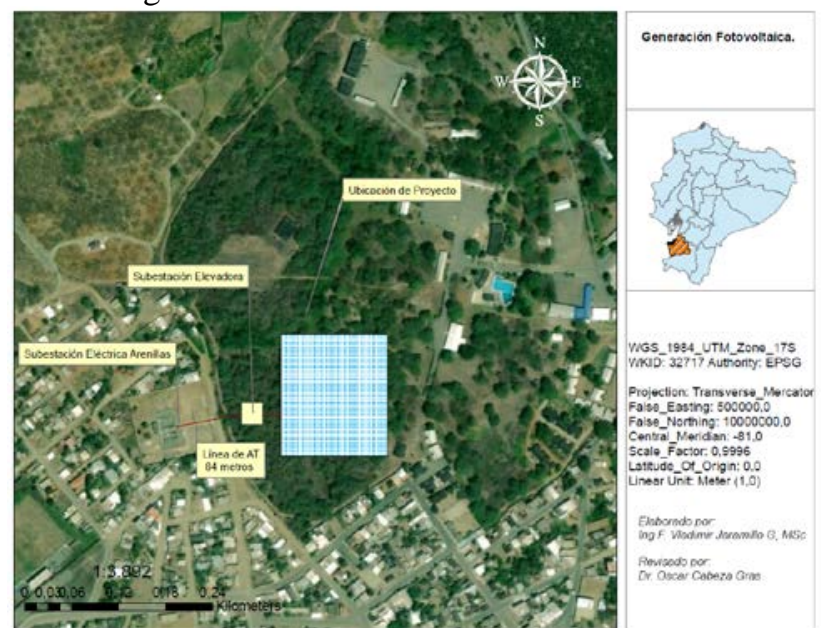

Figure 12. Connection between the solar plant and the Arenillas electrical substation.

By using a known electrical simulation software, we make a modulization of the photovoltaic solar plant and of the electrical distribution system of the substation, being the obtained results shown in Figure 13. Then we analyse the charge flux to determine the changes induced in them by the operation of the solar plant, mainly referred to the 69 $\mathrm{kV}$ bar of the Arenillas substation, which are reflected in Table II.

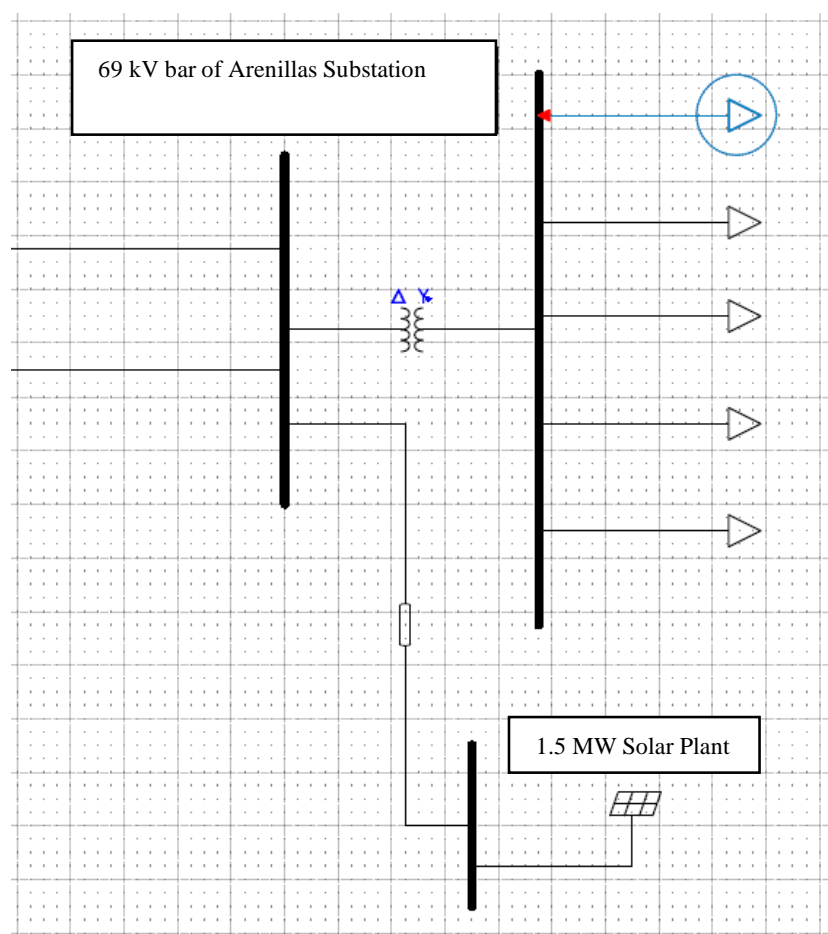

Figure 13. Simple wire diagram of the electrical connection between the solar plant and the Arenillas electrical substation

Table II. Bar voltage before and after the solar generation.

\begin{tabular}{|l|c|c|c|}
\hline $\begin{array}{l}\text { ARENILLAS } \\
\text { SUBSTATION }\end{array}$ & $\begin{array}{c}\mathrm{V} \\
(\mathrm{kVLL})\end{array}$ & $\begin{array}{c}\mathrm{V} \\
(\mathrm{pu})\end{array}$ & $\begin{array}{c}\% \\
\text { Reg }\end{array}$ \\
\hline $69 \mathrm{kV}$ barr without & 61,41 & 0,89 & $11,00 \%$ \\
\hline $69 \mathrm{kV}$ barr with & 67,41 & 0,98 & $2,30 \%$ \\
\hline
\end{tabular}

\section{Economic analysis}

In this section we develop an economic analysis of our project to demonstrate its viability. Thus, we estimate that the investment of about 1.5 \$ per watt installed, which is the average among minimum $1.3 \$$ for residential areas to a maximum of 1.69 \$ given recently for a more complicated project [8]. This prize implies an average total investment of 2.25 million USD (and a maximum of 2.535 million USD) to install the solar plant in operation, apart from the site prize. In the other side, actually in Ecuador the prize paid for a photovoltaic generated $\mathrm{kW} \cdot \mathrm{h}$ is regulated, an equals 0.5204 USD, with an annual increase of a $0.5 \%$.

Thus, taking into account that the annual production estimated is $2,419,708 \mathrm{~kW} \cdot \mathrm{h}$, the annual income would be $1,265,218$ USD the first year, and so the accumulated income along the 20 years (estimated as the effective life of the solar plant) would be 25,304,353 USD, with a cot of maintenance and operation estimated in 27,880 USD the first year. To calculate the total production of the solar plant, we have been aware of the solar panel degradation with time (about a $0.5 \%$ per year). Also, when calculating the total income, we have considered a discount rate of about $3 \%$, but also the increase in the prize paid for the energy produced (a $0.5 \%$ as commented) and the maintenance and operation cost (increase estimated in a 2 $\%$ annual). Thus, the calculated income for the first year would be $1,237,338$ and about $1,231,486$ the $20^{\text {th }}$ year. To resume this section, in Figure 14 we show the cash flow 
diagram of the investment obtained in the solar plant presented. As observed, the return period is only 22 months with a very good rentability, as big as $55.2 \%$, along the solar plant full operation life, which means a profit of about 15 million USD during the useful life of the photovoltaic power plant (estimated in 20 years).

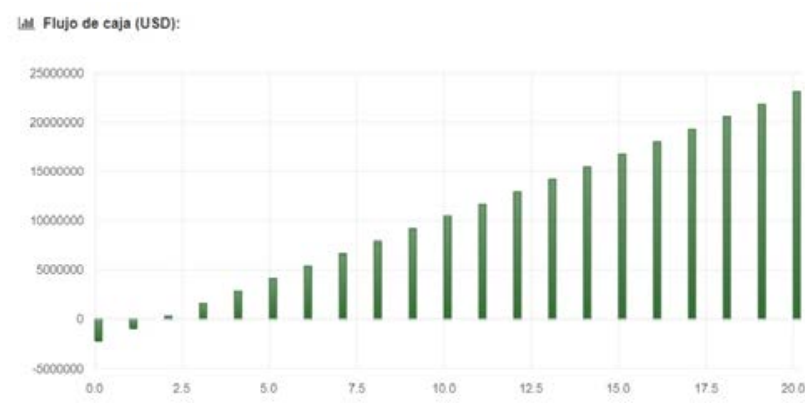

Figure 14. Cash Flow for the investment along the estimated life of the solar plant.

\section{Conclusion}

The geo-localization, physical characteristics of the site chosen to install the 1.5 MW photovoltaic solar plant, and the sunny weather all months of the year, are all optimal parameters. In addition, the site chosen is very near an existing electrical substation of the distribution network, which guaranties an easy connection with the consumers.

The estimated cost of the investment is about 2.25 million USD, being the return period of only 2 years, and an overall income of 25.3 million USD during the 20 years period of the plant life, with a rentability as big as 55,2 \%. In those estimations, we have taken into account an inflation of $2 \%$ and a discount rate of $3 \%$ per year. It is important to note the tons of GHG saved, and the strengthening of the electrical system in El Oro province of Ecuador. This last will translate in the improvement of the quality of the electricity system, which will facilitate the industrial and social development in the area.

\section{References}

[1] G. Price, Renewable power and energy_ photovoltaic systems, Momentum Press Engineering, New York (2019), pp. 33-34.

[2] Y. Yongheng, Advances in grid-connected photovoltaic power conversion systems, Woodhead Publishing, Ulsan (2019), pp. 21-25.

[3] T. Pavlovic, The Sun and Photovoltaic TechnologiesSpringer, Nis (2020), pp. 130-139.

[4] CENACE, real time operative information, (2019). www.cenace.org.ec

[5] A. Keyhani, Design of Smart Power Grid Renewable Energy Systems, Wiley-Blackwell, New York (2019), pp. 177-234.
[6] R. Precup, T. Kamal, Solar Photovoltaic Power Plants_ Advanced Control and Optimization Techniques, Springer, Chongqing (2019), ppl 72, 192,

[7] J. Stoustrup, A. Annaswamy, A. Chakrabortty, Z. Qu, Smart Grid Control__ Overview and Research Opportunities, Springer Internat, New York (2019), pp. 14, 23, 61.

[8] J.P. Muñoz-Vizhñay, M.V. Rojas-Moncayo, C.R. BarretoCalle, Incentive pertaining to energy the generation distributed in Ecuador. Ingenius. (2017). Vol. 19, pp. 60-68. 